\title{
El proceso de prueba en el espacio de trabajo geométrico: profesores en formación inicial
}

\section{The process of proof in the geometric work space: initial training teachers}

Elizabeth Montoya Delgadillo

Instituto de Matemática

Pontificia Universidad Católica de Valparaiso, Chile

emontoya@ucv.cl

RESUMEN • La enseñanza-aprendizaje de la demostración suele aparecer como objetivo explícito del currículo del liceo; sin embargo, no ocurre lo mismo en el currículo universitario de formación (inicial) de profesores, esto es, la enseñanza-aprendizaje de la demostración no es considerada objeto matemático sujeto a transposición. Utilizamos la Teoría de los Paradigmas y Espacio de Trabajo Geométrico para analizar, en profesores debutantes chilenos, los obstáculos que se generan debido a que los roles y el estatus de la demostración en la institución liceo difieren de los correspondientes en la universidad como institución formadora de profesores, para evidenciar concepciones geométricas y la carencia de transposiciones frente al proceso de prueba en Geometría. Esto último manifiesta una ruptura epistemológica, didáctica y cognitiva que suele encontrarse en la enseñanza de la Geometría.

PALABRAS CLAVE: paradigma; espacio de trabajo geométrico; proceso de prueba; didáctica de la Geometría.

ABSTRACT • Teaching and learning of proof often are explicit aims of the high school curriculum. Nevertheless, the same does not happen in the teachers' training curricula; i.e., neither teaching nor learning of proof are considered to be mathematical objects subject to didactic transposition.

Here we use the Theory of Paradigms and Geometric Work Space to analyze, in novel Chilean teachers, obstacles that generate because the roles and status of the proof in the institution high school differ from those in the university as a teachers' training institution. Thus, we give evidence of geometric conceptions and the lack of transpositions facing the process of proof in geometry. This in turn manifests an epistemological, didactic and cognitive break usually found in the teaching of geometry.

KEYWORDS: paradigm; geometric work space; process of proof; didactics of Geometry.

Fecha de recepción: noviembre 2012 • Aceptado: marzo 2014 


\section{INTRODUCCIÓN}

En el discurso escolar de la universidad, hacer demostraciones es con frecuencia considerado una práctica natural de los estudiantes de Matemáticas; tiene un status de herramienta de trabajo pues ellos la necesitan y utilizan en sus cursos de acuerdo a unas técnicas, un lenguaje y rigor propios de las diferentes teorías matemáticas. Sucede allí también que la demostración, al ser considerada una práctica o un saber hacer, no se enseña explícitamente y, por ello, pasa a ser una noción paramatemática (Chevallard, 1985). En otras instituciones escolares, como el liceo, la demostración tiene un estatus de noción matemática, y el profesor tiene el deber de enseñarlas, hacerlas y solicitarlas a sus alumnos. Luego cabe preguntarse ¿cómo puede un profesor debutante transponer este saber si él mismo no ha tenido este proceso en su formación inicial?.

Nuestro objetivo fue estudiar la articulación que realiza un profesor debutante «frente a un objetivo de enseñanza en términos de un saber enseñado» (ibíd.) en torno al proceso de prueba en el que se inserta la demostración (Balacheff, 1987). Dado que la transposición de saberes se realiza tras la formación inicial, estudiamos el doble pasaje y doble rol entre dos instituciones escolares: el primero, de alumno de liceo a estudiante universitario, y el segundo, de este último a profesor de liceo.

Analizamos al estudiante que realiza la práctica profesional -de ahora en adelante el estudianteprofesor, E-P-, quien a la vez es un profesor en el liceo y un estudiante en la universidad; este doble rol nos es propicio para conocer el estatus que le confiere a las pruebas que se exigen y elaboran en Geometría. Postulamos tres rupturas, ejes de nuestra investigación, relativas a la enseñanza-aprendizaje de la demostración en Geometría, que provienen de los obstáculos (Brousseau, 1983) epistemológico, didáctico y cognitivo respectivamente.

\section{PLANTEAMIENTO DEL PROBLEMA}

\section{El currículo en el liceo y en la universidad}

Siguiendo a Balacheff (1997), distinguimos prueba, una justificación cuya validez puede ser modificable, de demostración, una prueba que adopta una forma particular y lógica y se apoya en conocimientos institucionalizados (ver 3.3, más adelante).

Diversas investigaciones muestran las dificultades que los estudiantes de primer año universitario manifiestan respecto a la demostración en Matemáticas; ellas fueron clasificadas por Moore en 1994 en «desconocimiento de las definiciones, dificultad para elaborar ejemplos incluso si esos son simples, dificultad en iniciar pruebas y dificultad relativa al lenguaje» (cf. Gueudet, 2005: 166).

La cultura escolar no ofrece criterios de validación de un argumento que conforma una demostración. El contrato didáctico (Brousseau, 1998) en torno al proceso de prueba en la enseñanza universitaria es poco claro, específicamente en lo que se refiere a las exigencias en la elaboración de una demostración y en la no uniformidad de criterios válidos en su conformación. En efecto, allí la práctica de la demostración es considerada una herramienta de trabajo para los estudiantes de Matemáticas, quienes normalmente deben utilizarla para tener éxito en sus cursos y deben así responder ante exigencias en su elaboración. La demostración no es un saber explícito de enseñanza en el currículo universitario, a pesar de ser una prueba privilegiada en la institución (como constataremos posteriormente).

Por otro lado, que un formador universitario exhiba cómo se demuestra no es suficiente para afirmar que haya enseñado a demostrar, y así la demostración pasa a ser parte de un currículo ignorado (Castela, 2005) por esa institución escolar. El estudiante en formación replica lo realizado por su profesor formador, esto es, sigue pasos y desarrolla técnicas para realizar un buen camino de deducción mientras se nutre de un cuerpo de conocimientos matemáticos. En el aprendizaje de la demostración, 
ambos actores, profesor formador y estudiante universitario, comparten un contrato didáctico en el que la imitación sería la principal característica.

A lo anterior se suman, en un ámbito no geométrico, los resultados de Durand-Guerrier y Arsac (2003), quienes concluyen que no hay gran diferencia entre la demostración desarrollada por el profesor en clase de Matemáticas superiores y la de la investigación que publica un matemático profesional.

En la institución formadora donde realizamos nuestra fase de experimentación, una buena parte de estudiantes de Matemáticas se forman como profesores para la enseñanza media (secundaria) de Chile. Estos deberán hacer, enseñar y solicitar demostraciones a sus alumnos a partir del primer año de enseñanza media (14-15 años), y deben promover el razonamiento argumentativo y deductivo. Sin embargo, hemos podido constatar, por un lado, que en la universidad no existe reflexión sobre la transposición didáctica de la demostración y, en general, del proceso de prueba, y que la responsabilidad de transponer estos saberes como objetivo de enseñanza queda bajo la responsabilidad del profesor en formación y no de la institución formadora. Luego nos preguntamos cómo articulará este saber el profesor debutante; para ello, es pertinente conocer el estatus que él le otorga al proceso de prueba y cuál es su concepción de demostración.

\section{La demostración en un espacio de trabajo matemático}

El cómo y para qué se usa la demostración dependen del rol que le asigna el individuo en relación con la institución a la que pertenece; el rol que cumple para el matemático profesional es distinto para el mismo individuo en cuanto profesor. Hemos observado, además, que la concepción de demostración que tiene el profesor varía según las instituciones escolares (Montoya, 2010). Al respecto, De Villiers (1993) reconoce la existencia de cinco funciones de la demostración: verificación, explicación, sistematización, descubrimiento y comunicación, y critica el enfoque parcial que se le ha otorgado como medio de verificación al ser habitualmente usada por los profesores de Matemáticas para convencer, debiendo ser la convicción un prerrequisito para demostrar y debe en todas ellas, la demostración, ser presentada como una actividad significativa para los estudiantes.

Problemáticas asociadas a la argumentación y demostración han sido objeto de estudio para diferentes investigadores, entre otros, Balacheff (1987), Boero (1999), Duval (1991; 1992; 1995), Plantin (1996), Toulmin (1958), Ducrot (1972), Godino y Recio (2001). Aquellas han sido trabajadas principalmente desde perspectivas cognitivas, lingüísticas y epistemológicas. En estos trabajos, hay concepciones fundadas de lo que significa una demostración en Matemáticas y de los razonamientos que ello involucra; también se evidencia la importancia de realizar demostraciones en el aula y las dificultades que trae consigo. La vigencia de estas problemáticas se puede apreciar, en particular, en el ICMI Study 19: Proof and Proving in Mathematics Education (2009), en el que se evidencia la preocupación por desarrollar una cultura frente a la demostración en el aula en todos los niveles educativos.

Concebimos la demostración en la universidad como una noción paramatemática y en el liceo como una noción matemática.

Por otro lado, podemos observar que diversos significados le son asociados a las palabras demuestre, pruebe y justifique, que podrían perecer sinónimas; sin embargo, no siempre lo han sido en la historia de las Matemáticas (Arsac, 1987), y el razonamiento asociado a la demostración ha evolucionado en términos epistemológicos, tanto en su discurso como en la significación que conlleva su práctica (Barbine, 1989). Más aún, Hanna $(1995 ; 2000)$ afirma que estarían surgiendo desde las propias Matemáticas nuevas estrategias de validación que desafían la concepción clásica de la demostración deductiva, basadas en comprobaciones experimentales, desarrolladas mediante ordenadores y en las que el rol de la visualización es una contribución potencial (ibíd., 2007). Estas nuevas estrategias de validación difieren del propio trabajo matemático, que describe la prueba en la práctica real del matemático como 
un «argumento convincente, juzgado como tal por jueces calificados» (Hersh, 1993: 389). Esta idea de demostración - deductiva, de modo rígido y absoluta- no debe ser la única concepción posible y sus significados dependen de las instituciones escolares (Godino y Recio, 2001). Así, podemos afirmar que el concepto de demostración es una cuestión paradigmática -en el sentido de Kuhn (1962 y 1971) - y, en nuestro caso, que depende de la institución escolar.

\section{Pasajes y rupturas en torno a la demostración}

Llamamos primer pasaje al cambio de institución, entre liceo (de 14 a 18 años) y universidad, que convierte al alumno en estudiante universitario; este necesitará de la validación bajo una posición teórica y formal, al verse enfrentado a esta y otras exigencias institucionales que debería aprender para tener éxito en sus cursos de Matemáticas.

La evidencia muestra que para muchos estudiantes será realmente el debut de hacer demostraciones y aun de cualquier tipo de prueba, ya que sus profesores del liceo (Araya, 2008) no hacen demostraciones ni razonamiento deductivo en sus clases. Por otra parte, el informe del Sistema de Medición de la Calidad de la Educación (SIMCE 1 , 2003, 2007, 2009) da evidencias de la baja cobertura curricular en el eje de Geometría -eje propicio para enseñar a demostrar-, y de correlación entre los contenidos en los que los profesores se sienten menos preparados y aquellos en que los estudiantes tienen menos éxito. En este eje temático (y en otros), se aprecia que la práctica de la demostración es débil en el liceo. Menares (2009) y Henríquez (2009) muestran el estatus de la demostración en textos escolares y su concepción por parte de profesores de liceo.

El segundo pasaje convierte al estudiante universitario (de Pedagogía) en profesor en el liceo, pasando así a ser el principal responsable de articular sus saberes en torno al proceso prueba (y a las demostraciones) respondiendo al currículo esperado en el liceo; esto es, debe hacer, solicitar y enseñar a demostrar. Pero cabe preguntarse ¿cómo lo hace si en la universidad no ha tenido un espacio explícito para transponer este saber? Cuando realiza su práctica profesional, el E-P debe obedecer a dos exigencias institucionales bajo el rol de estudiante y bajo el rol de profesor.

Considerando que la demostración es un saber hacer, postulamos que existen rupturas en estos pasajes -en torno a este saber- y que se originan por la presencia de obstáculos de índole cognitivo, didáctico y epistemológico. El de origen cognitivo se manifiesta en la producción misma de E-P que se enfrenta a la enseñanza de la demostración; el epistemológico se refiere a la concepción de la demostración (geométrica) en el desarrollo de las Matemáticas; y el didáctico se refiere a la concepción de la enseñanza de la demostración tanto en el liceo como en la universidad. Es decir, estos obstáculos son relativos a la epistemología tanto de las Matemáticas como del profesor.

Ese doble pasaje que convierte en un estudiante en la universidad y luego en un profesor en el liceo nos es propicio para conocer el estatus que él le da a las pruebas que se exigen y elaboran en Geometría; para ello, pasamos a exponer el marco teórico que sustenta esta investigación.

\section{FUNDAMENTOS TEÓRICOS}

El cuerpo teórico que sustenta nuestro trabajo es el de los Paradigmas geométricos y el Espacio de Trabajo Geométrico (ETG) desarrollado por Houdement y Kuzniak (1999, 2000, 2006) y Kuzniak (2004). Los autores consideran la naturaleza del trabajo geométrico cuando un geómetra ${ }^{2}$ se enfrenta a una tarea en Geometría. Además, consideramos la Tipología de prueba de Balacheff (1987) confron-

1. Evaluación a la que se someten los estudiantes de 10, 13 y 15 años del sistema educativo en Chile

2. Geómetra es aquí la persona que enfrenta una tarea en Geometría, ya sea un investigador, un profesor o un estudiante 
tándola con la noción de razonamiento y demostración de Duval (1995). Estas teorías nos permiten hablar del proceso de prueba involucrado en el ETG del geómetra como en el de las instituciones escolares involucradas.

La teoría posee dos grandes ejes: los Paradigmas y el ETG. En la actualidad, la teoría considera un espacio de trabajo matemático global, ETM, que depende del dominio matemático -así, el ETG es ahora $\mathrm{ETM}_{\mathrm{G}}$, y se tiene además $\mathrm{ETM}_{\mathrm{A}}$, etcétera- y los paradigmas se pueden interpretar como la caracterización del ETM (Kuzniak, 2011).

\section{Los paradigmas geométricos}

Los paradigmas geométricos se configuran considerando tres ejes: lo filosófico (creencias de la comunidad), lo cognitivo (modos de pensamiento -intuitivo, deductivo, experimental-) y lo epistemológico (geometría en su evolución). Así, se plantea la existencia de tres tipos de geometrías o paradigmas geométricos provistos cada uno de un ETG que pasamos a explicitar.

- Geometría natural (GI). Refleja la existencia de una relación con la realidad; los objetos están definidos por el modelo geométrico pero en correspondencia con la realidad espacial y local del individuo. Los medios de prueba son de tipo material y se utilizan artefactos para la representación del objeto (no abstracto sino concreto). El modelo geométrico subyacente es la idea que el individuo tiene y se forja de la Geometría euclidiana.

- Geometría axiomática natural (GII). La geometría es concebida como el esquema de la realidad. El razonamiento de validación se funda sobre las leyes hipotéticas deductivas del sistema axiomático en juego (propiedades, definiciones, etcétera). El modelo geométrico es local, puesto que los problemas para ser resueltos no requieren de la presencia de todos los axiomas.

- Geometría axiomática formalista (GIII). Los objetos geométricos provienen de una axiomática elegida con toda la rigurosidad y el formalismo del modelo geométrico elegido. El razonamiento de validación se realiza exclusivamente a través del sistema formal de axiomas del modelo geométrico subyacente. Este paradigma surge con la aparición de las geometrías no-euclidianas, pero incluye la euclidiana.

Si se está trabajando en una Geometría no euclidiana, el geómetra evidencia naturalmente la presencia de todos los axiomas ante cualquier demostración; en el caso de la euclidiana, es más difícil distinguir los dos tipos de geometrías.

\section{El Espacio de trabajo geométrico $\left(\mathrm{ETG}_{\text {o }} \mathrm{ETM}_{\mathrm{G}}\right)$}

El ETG es el ambiente en el cual se concibe la reflexión fruto de la interacción entre un individuo y los problemas geométricos, es un ambiente organizado por y para el geómetra (Kuzniak, 2004) mediante la articulación de dos planos: el epistemológico y el cognitivo.

El plano epistemológico está compuesto por tres polos, a saber: el referencial, en el que se explicita el modelo geométrico, el espacio local y real, y los artefactos. El plano cognitivo se compone de los polos visualización, construcción y prueba.

Los planos se articulan mediante tres génesis como se observa en la figura 1, en los que se muestra un ETM global, es decir, sin considerar un dominio específico de las Matemáticas. Por ello, el espacio local y real es llamado representamen (o representante). 


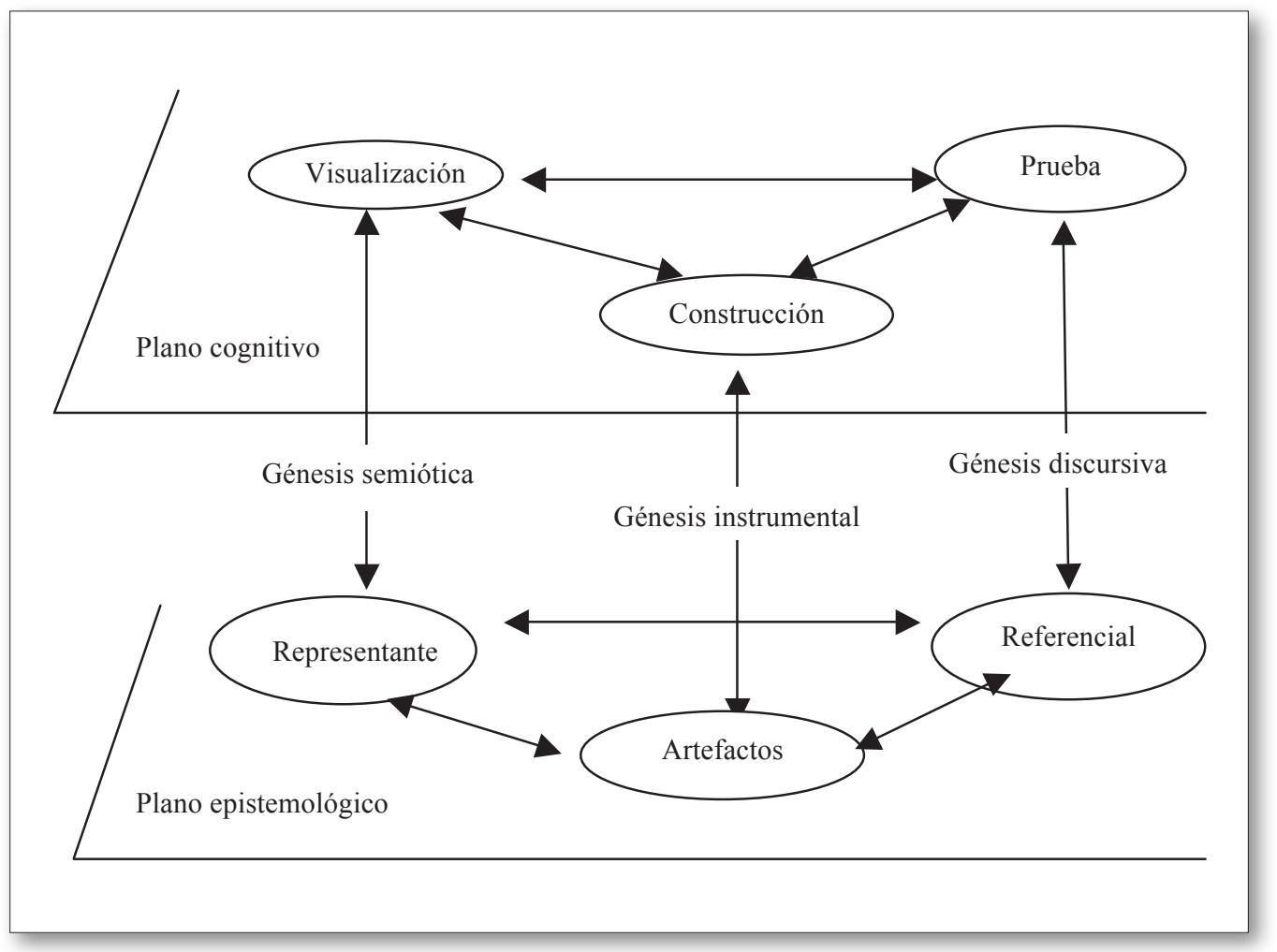

Fig. 1. ETM en el (Kuzniak, 2011).

Dependiendo de la función y de la reflexión del geómetra cuando se enfrenta a un problema geométrico, hay tres tipos de ETG; estos son:

- ETG de referencia: el espacio de trabajo definido de manera ideal en función de criterios matemáticos. Se dice que el utilizador es un experto epistémico. Se puede considerar como el ETG institucional de la comunidad de los matemáticos. Un utilizador natural de este ETG es un investigador en Geometría.

- ETG idóneo: el espacio en el que se concibe la reflexión sobre la reorganización didáctica de los componentes, es decir, está definido en términos didácticos. Un utilizador de este ETG es el profesor.

- ETG personal: el espacio definido por un geómetra, fruto de la reflexión entre los conocimientos aprendidos y los puestos en práctica, de acuerdo a sus conocimientos y capacidades cognitivas. Utilizadores naturales de este ETG son el estudiante y el profesor.

El ETG es un sistema dinámico en el que se articulan los componentes de ambos planos, en el que se evidencian distintas relaciones entre estos polos - llamadas circulaciones del ETG- que dependen de la tarea geométrica que moviliza el geómetra.

Nos centraremos, particularmente, en el polo prueba para estudiar al E-P en sus distintos roles, ya que para abordar la demostración él requiere articular los elementos del espacio local y real de acuerdo al modelo geométrico del referencial, y eventualmente utilizará el polo visualización. Esto nos dice que los componentes del plano cognitivo y epistemológico no solo se relacionan «uno a uno», como se podría leer de la figura 1, sino que al contrario, en la actualidad se habla de una relación de planos verticales en el ETM. 


\section{El razonamiento y la tipología de prueba}

La tipología de prueba (Balacheff, 1997) permite analizar las fuentes de validación que el geómetra utiliza. Para él, prueba designa sobre todo una justificación cuya validez puede modificarse con arreglo a la experiencia de la comunidad y del tiempo en un contexto social -lo que se vincula con la visión paradigmática de Kuzniak-; demostración es la prueba que adopta una forma particular y lógica, y se apoya en un cuerpo de conocimientos fuertemente institucionalizados: conjunto de definiciones, de teoremas y reglas de deducción cuya validez es compartida por la comunidad matemática.

La concepción de rigor matemático es un implícito que depende de la institución escolar, y su apropiación por parte de los alumnos requiere de una construcción cognitiva especial, no espontánea, y que debería ser enseñada.

La concepción de demostración de Balacheff (1987) es compatible con la noción de Duval (1991; 1992). La diferencia más sustancial radica en la concepción de razonamiento. Para el primero, una explicación es un argumento considerado como un razonamiento; para el segundo, una explicación no siempre es un razonamiento, pues su valor epistémico en las proposiciones en las que interviene puede ser semántico y no lógico, debido a que en general una explicación se preocupa del contenido por encima del hecho de modificar el enunciado-objeto (Duval, 1995). En nuestra investigación, consideramos la concepción de Balacheff.

Balacheff distingue dos tipos (o grupos) de pruebas.

Las pruebas pragmáticas - para decidir- están ligadas a la acción y a la experiencia, hay presencia de saberes prácticos, las justificaciones se realizan a través de objetos materiales concretos o de su representación. En este grupo se distinguen: empirismo ingenuo, experiencia crucial, y ejemplo genérico.

Las pruebas intelectuales - para saber- provienen de una forma particular de razonar: se articulan argumentos o cadenas de argumentos con una clara producción hacia el lenguaje simbólico; hay un pasaje a lo algebraico, se dejan de lado los objetos materiales y su relación con la experiencia material. En este grupo se distinguen: experiencia mental, demostración, y cálculo sobre el enunciado.

La figura 2 muestra la articulación de estas herramientas teóricas; en ella se ve el tipo de pruebas asociadas a cada paradigma geométrico en los que habita el E-P. Proponemos que en el ETM existen pruebas y razonamientos de validaciones en concordancia con los paradigmas geométricos: el empirismo ingenuo, con el que se puede manipular a través de un solo caso una propiedad, es característico de un trabajo en GI; la demostración es trabajo clasificable en GII o en GIII; sin embargo, y tal como se ve en el diagrama, hay otras pruebas (ejemplo genérico) que pueden ser clasificadas en GI o en GII (incluso en GIII) dependiendo de la producción del geómetra. La experiencia crucial se caracteriza porque la validación toma en cuenta la problemática de la generalidad y la «resuelve» mediante el uso de un caso particular que reconoce como «no especial». La experiencia mental hace referencia a cuando la persona se independiza de la representación del objeto, se observa un guión que no tiene necesariamente la estructura de una demostración, no se observa un trabajo de deducción. 


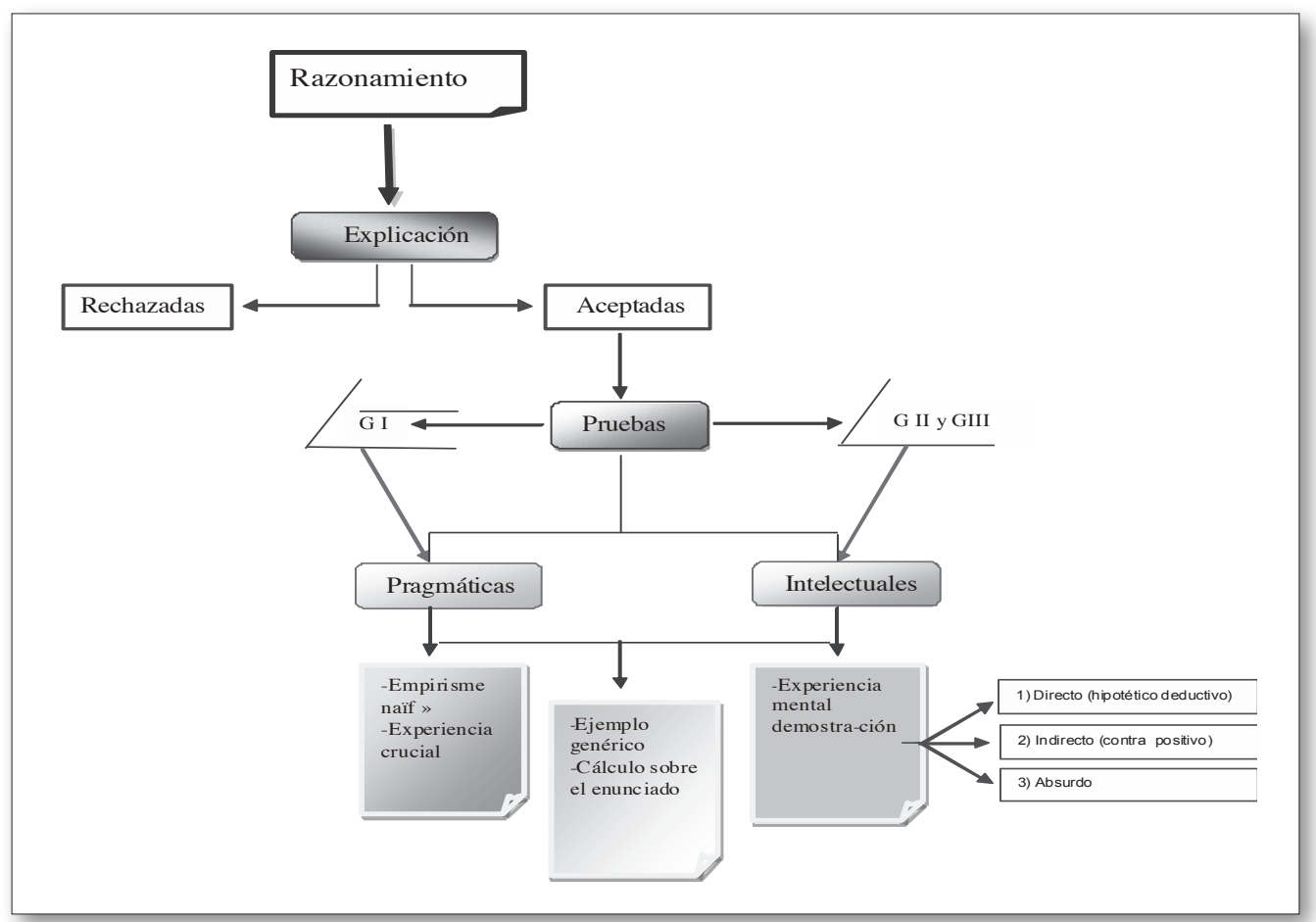

Fig. 2. Tipología de pruebas y los paradigmas geométricos.

\section{METODOLOGÍA}

Para estudiar ETG-personal del E-P de acuerdo con su rol, necesitamos saber cómo fue formado. Como se ilustra en la figura 3, se utilizaron distintos instrumentos que nos permitieron dar información del ETG-personal del E-P. Las flechas (directas) que van del ETG-idóneo y ETG-referencia hacia el ETG-personal indican la influencia de estos espacios de trabajo en este último. Para estudiar el ETGreferencia se utilizaron programas (currículos) y entrevistas a formadores universitarios y de liceo. Para el ETG-idóneo de la universidad, se consideraron como insumos las evaluaciones finales (exámenes) y el cuaderno de Geometría de primer año y las entrevistas antes señaladas. Finalmente, para el ETGpersonal del E-P, se aplicó un cuestionario a 19 E-P de noveno semestre (último año) y una encuesta a 25 estudiantes que habían reprobado el curso de Geometría (primer año).

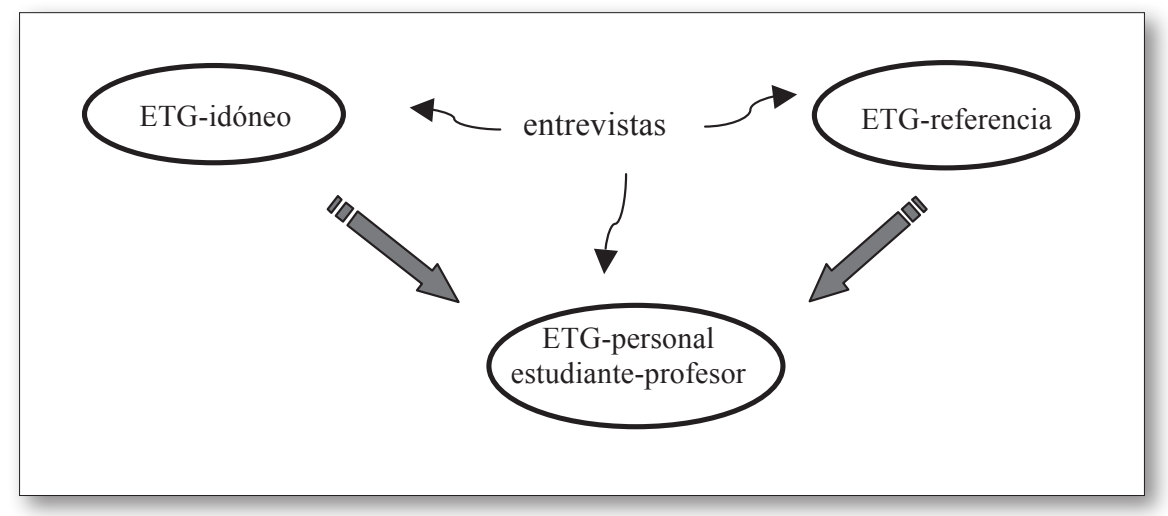

Fig. 3. Insumos metodológicos. 
El ETG-referencial varía dependiendo del utilizador y de dónde está inserto; por ello, se tomaron decisiones metodológicas sobre los insumos requeridos. Por este motivo, se consideraron los programas del Ministerio (currículo) para el liceo (MINEDUC) y, en el caso de la universidad, los programas de los cursos de Geometría (currículo) y las entrevistas a profesores formadores.

Para el ETG-idóneo, definido en términos de la organización didáctica, se consideraron para la universidad otro tipo de entrevistas y el análisis didáctico del curso de Geometría a través del cuaderno del estudiante; además, cinco evaluaciones finales (exámenes) correspondientes a un período de cuatro años (2003-2006) y el cuaderno en su dimensión teórica y práctica (ver tabla 1).

En el liceo, estudiamos los textos escolares y el programa de Geometría (bajo el rol de las sugerencias de enseñanza que entregaba) centrados en el proceso de prueba.

Las entrevistas, de carácter semiestructurado, abordaron cuatro ejes: ambiente profesional (rol de formador), artefactos, proceso de prueba y enseñanza de la demostración. Se consideraron cuatro a profesores formadores de la universidad, una a un profesor formador del liceo y una a un profesorayudante (profesor debutante). Los formadores fueron elegidos principalmente por la experiencia (de 15 a 25 años de docencia) que tienen en sus respectivas instituciones.

Tabla 1.

Configuración del estudio del curso de Geometría de primer año

\begin{tabular}{|l|l|}
\hline \multicolumn{1}{|c|}{ Tipo de sesión } & \multicolumn{1}{c|}{ Insumo } \\
\hline 1. Sesión teórica & Cuadernos de Geometría \\
\hline & \\
\hline 2. Sesiones prácticas & \\
2.1 Taller sala de clase & Guía sala de clases \\
2.2 Taller sala de laboratorio & Guía laboratorio y proyectos \\
\hline
\end{tabular}

El ETG personal del E-P fue realizado a través de un cuestionario tomado a 19 estudiantes en práctica profesional. El cuestionario aborda dos roles, el de profesor y el de estudiante. Las preguntas se diseñaron para hacerlo transitar en los recorridos (posibles) del plano epistemológico y del plano cognitivo del ETG. Además, se aplicó una encuesta a 25 estudiantes de primer año del curso de Geometría para identificar elementos de la ruptura en el primer pasaje (liceo-universidad).

Con las herramientas teóricas y considerando los insumos metodológicos, reformulamos nuestros interrogantes iniciales para analizar el ETG de referencia de la institución. P1: ¡cuál es el paradigma geométrico dominante en la formación inicial de profesor de Matemáticas? P2: para conocer el ETG personal del E-P en relación con el plano cognitivo, ¿cuáles son aquellos argumentos que le son válidos en la conformación de una demostración? En términos generales, P: ¿cómo un futuro profesor considera las pruebas en Geometría, es decir, ¿qué argumentos le son válidos en diferentes roles e instituciones?

\section{ALGUNOS RESULTADOS}

\section{Una aproximación al ETG idóneo del formador}

\section{Curso de Geometría: las evaluaciones}

Entre las evaluaciones, los exámenes nos permiten estudiar las pruebas exigidas (solicitadas) a los estudiantes de primer año en el curso de Geometría. En ellas aparecen contenidos y exigencias del curso en cuestión. Las cinco evaluaciones que corresponden a exámenes de 2003 a 2006 se resumieron bajo la 
tarea solicitada en cada año y su frecuencia, descritas con la consigna del verbo de acción. Las pruebas solicitadas quedan registradas como justifique, verifique y demuestre y, como se ve en la tabla 2, la prueba privilegiada es la demostración. De esta forma, el estudiante debe poner en juego el polo referencial teórico (del ETG-idóneo) que domina ${ }^{3}$ al final del curso de Geometría de primer año universitario.

Tabla 2.

Examen a estudiantes de primer año del curso de Geometría

\begin{tabular}{|l|c|c|c|c|c|c|}
\hline \multirow{2}{*}{\multicolumn{1}{c|}{ Tareas }} & \multicolumn{7}{c|}{ Año } \\
\cline { 2 - 8 } & 2003 & 2004 & 2005 & 2006 & 2006 & Total \\
\hline Conjeture & 1 & 1 & -- & -- & -- & 2 \\
\hline Verifique & 1 & -- & -- & -- & -- & 1 \\
\hline Justifique & -- & -- & -- & -- & -- & 0 \\
\hline Demuestre & 3 & 7 & 5 & 6 & 2 & 23 \\
\hline Construya & 1 & -- & -- & -- & - & 1 \\
\hline Represente & -- & -- & -- & -- & 1 & 1 \\
\hline Calcule & -- & -- & -- & -- & 1 & 1 \\
\hline N.o Preguntas & 6 & $8(*)$ & 5 & $6(*)$ & $4(* *)$ & 29 \\
\hline
\end{tabular}

$(*)$ Los estudiantes deben elegir cinco preguntas. $\left({ }^{* *}\right)$ El profesor no es el mismo que el de los años anteriores

Se puede observar que el concepto de demostración en el ETG-personal de los estudiantes fue transado por el formador: la producción del estudiante corresponde a pruebas intelectuales del tipo experiencia mental; hay explicaciones (o guiones) en los que se manifiestan deducciones y, por lo general, están escritas en lenguaje mixto. En las pautas de corrección propuestas por el formador, se observa que las argumentaciones corresponden a demostraciones, es decir, este acepta como demostración las pruebas intelectuales del tipo experiencia mental desarrolladas por sus estudiantes, información que se triangula con la entrevista realizada al formador en Geometría.

De esta forma, con los elementos analizados, podemos afirmar que en las evaluaciones el paradigma geométrico asociado al ETG-personal que está presente es GII.

\section{Curso de Geometría: los talleres}

En el curso de Geometría de primer año se observan ejercicios con un alto énfasis en el demostrar, situación que es corroborada en la entrevista al formador universitario en Geometría (FUG1), en la cual este declara el interés y estatus que confiere a la demostración:

FUG1: En el taller (clase práctica) son puras demostraciones, también se hacen construcciones [...] las demostraciones son básicas, para que los estudiantes se acostumbren a demostrar, después este es un curso que le ayudará bastante en su formación universitaria.

Se observa que cohabitan dos paradigmas geométricos y varían dependiendo de la tarea que se solicita; en las sesiones teóricas, se observa a la vez GIII y GII, mientras que en las sesiones prácticas correspondientes al uso del software geométrico se observa GII y GI.

3. Contenidos geométricos que se espera que sean parte de sus conocimientos adquiridos (en francés maîtriser). 
En la tabla 3, se muestra el resumen del análisis de los trabajos solicitados en las guías que permiten desarrollar el taller usando un programa de Geometría dinámica. Esto corresponde al análisis de la parte práctica del curso de primer año. Las tareas clasificadas de las guías se resumen (tabla 3) de acuerdo a los componentes cognitivos asociados al ETG y, como se observa, solo se ha utilizado el verbo de la consigna de la tarea en cuestión. Cabe señalar que, debido a la naturaleza del software, el componente visualización siempre está presente, señalado con una $x$.

Tabla 3.

Guías de ejercicios con instrumentos no clásicos (Geometría dinámica)

\begin{tabular}{|c|c|c|c|}
\hline Tarea & $\begin{array}{c}\text { Artefacto } \\
\text { «Construcción» }\end{array}$ & $\begin{array}{l}\text { Referencial } \\
\text { «Prueba» }\end{array}$ & $\begin{array}{l}\text { Objeto real y local } \\
\text { «Visualización» }\end{array}$ \\
\hline \multicolumn{2}{|c|}{ Mida } & Conjeture & $\mathrm{x}$ \\
\hline \multicolumn{2}{|c|}{ Construya } & Pruebe & $\mathrm{x}$ \\
\hline \multicolumn{2}{|c|}{ Verifique } & Demuestre & $\mathrm{x}$ \\
\hline & & Verifique/Compruebe & $\mathrm{x}$ \\
\hline
\end{tabular}

Cuando el estudiante mide, construye o verifica (columna 1) lo hace con la interacción entre el polo artefacto y construcción del ETG en forma dinámica y sistemática. De manera análoga, hemos clasificado las otras consignas en los polos del plano epistemológico y cognitivo respectivamente. A nivel de los paradigmas, se observan en estas guías que cohabitan GI y GII, esto es, hay tareas propias de GI, como medir y verificar a través de instrumentos, pero también propias de GII, como la construcción.

A nivel de las pruebas, se solicita al estudiante que verifique, conjeture y pruebe sus conjeturas, que pruebe propiedades y también de forma explícita que demuestre, pero no se aclara cuáles serán las pruebas permitidas. Por ello, fue posible encontrar producciones de los estudiantes en GI, ya que se permiten las pruebas pragmáticas usando la comprobación por medio de la herramienta del programa (medir), como se puede apreciar a continuación (figura 4).

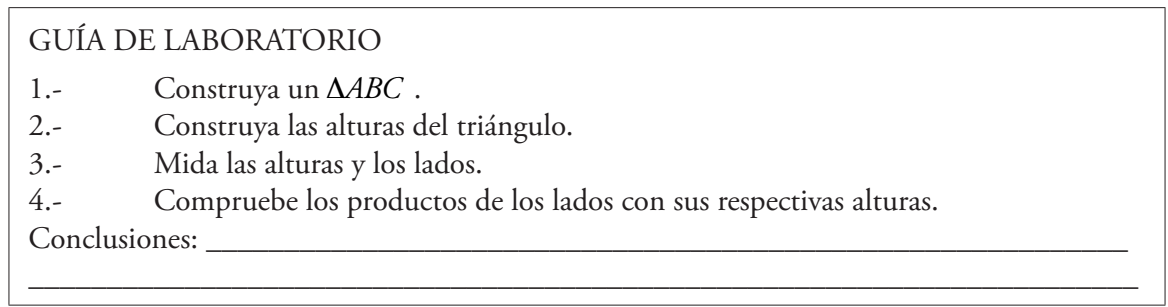

Fig. 4. Guía de laboratorio, taller.

En la guía está involucrado el teorema de las alturas en un triángulo, que es parte del programa de segundo medio en el liceo, y en la universidad parte del contenido de relaciones métricas y de incidencia en polígono. ${ }^{4}$ Sin embargo, en esta tarea nada se dice del tipo de triángulo involucrado, luego, a priori, las posibilidades de elección y análisis que debe realizar el estudiante son diferentes a las de este teorema en la enseñanza media.

La medición es un elemento clave para clasificar esta tarea en GI; la obtención de conclusiones y las posibles justificaciones están reguladas por el uso de la tecnología. En este ambiente de la Geometría dinámica, se privilegia el desarrollo de pruebas pragmáticas del tipo empirismo-ingenuo y ejemplo

4. Programa curso de Geometría primer semestre. 
genérico. Se establece un espacio de trabajo idóneo en el que los principales polos de circulación son artefacto-construcción-visualización y prueba.

El uso del software como instrumento geométrico no clásico posee otras dificultades cuando se compara con los instrumentos geométricos clásicos, pero la gran diferencia es la interacción que se tiene con la visualización -componente cognitivo asociado al ETG- al usarse la herramienta del movimiento que tiene incorporado el programa informático. Tal herramienta permite visualizar y experimentar de manera integrada y dinámica familias de dibujos asociados a una figura geométrica, pero no es evidente el trabajo expreso del polo prueba y referencial teórico por parte del estudiante.

A continuación mostramos una hoja de trabajo (figura 5a) de un grupo de estudiantes de este curso de primer año, cuya tarea es demostrar la congruencia de dos segmentos.

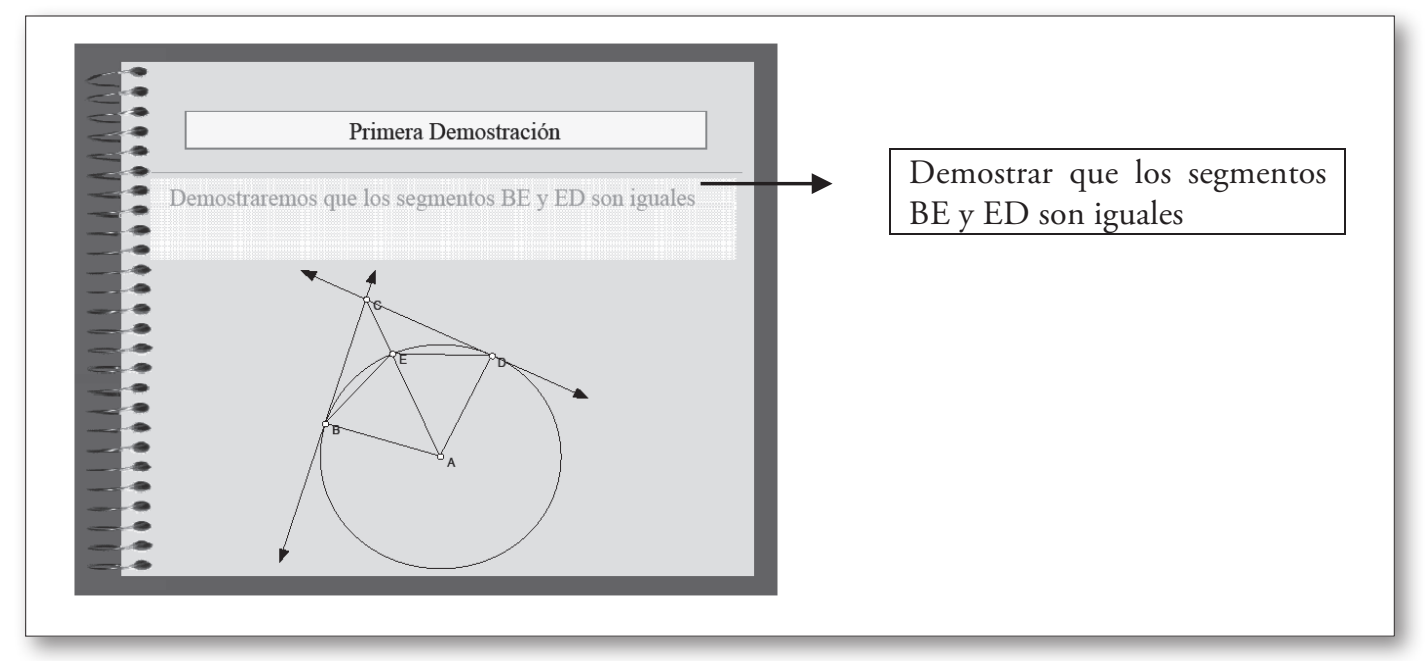

Fig. 5a. Proyecto Medida y Demostración

Es interesante observar en este trabajo que los estudiantes, de manera intencionada, han propuesto dos tipos de justificaciones:

- La medición de trazo y ángulo con un instrumento geométrico no tradicional: la figura geométrica fue construida y posteriormente se usó un instrumento de medición con la herramienta del programa.

- En la misma hoja, los estudiantes muestran las relaciones y propiedades que se cumplen en la incidencia.

En la primera justificación, se considera la manipulación del objeto y la medición tanto de trazos como de ángulos de un caso en particular, lo que permite afirmar que es una prueba pragmática del tipo empirismo-ingenuo y, por lo tanto, a nivel de GI. La segunda utiliza un lenguaje algebraico, débil probablemente por el uso del programa y las deducciones y, a pesar de que la figura es parte del desarrollo de la justificación, la clasificamos como demostración, es decir, una prueba intelectual y a nivel de GII.

En el ETG-personal de estos estudiantes, se observa que la lectura de los datos y el objeto geométrico siempre se desarrollan con el dibujo, es decir, la visualización juega un importante rol en ambas justificaciones (ver figura 5b). 


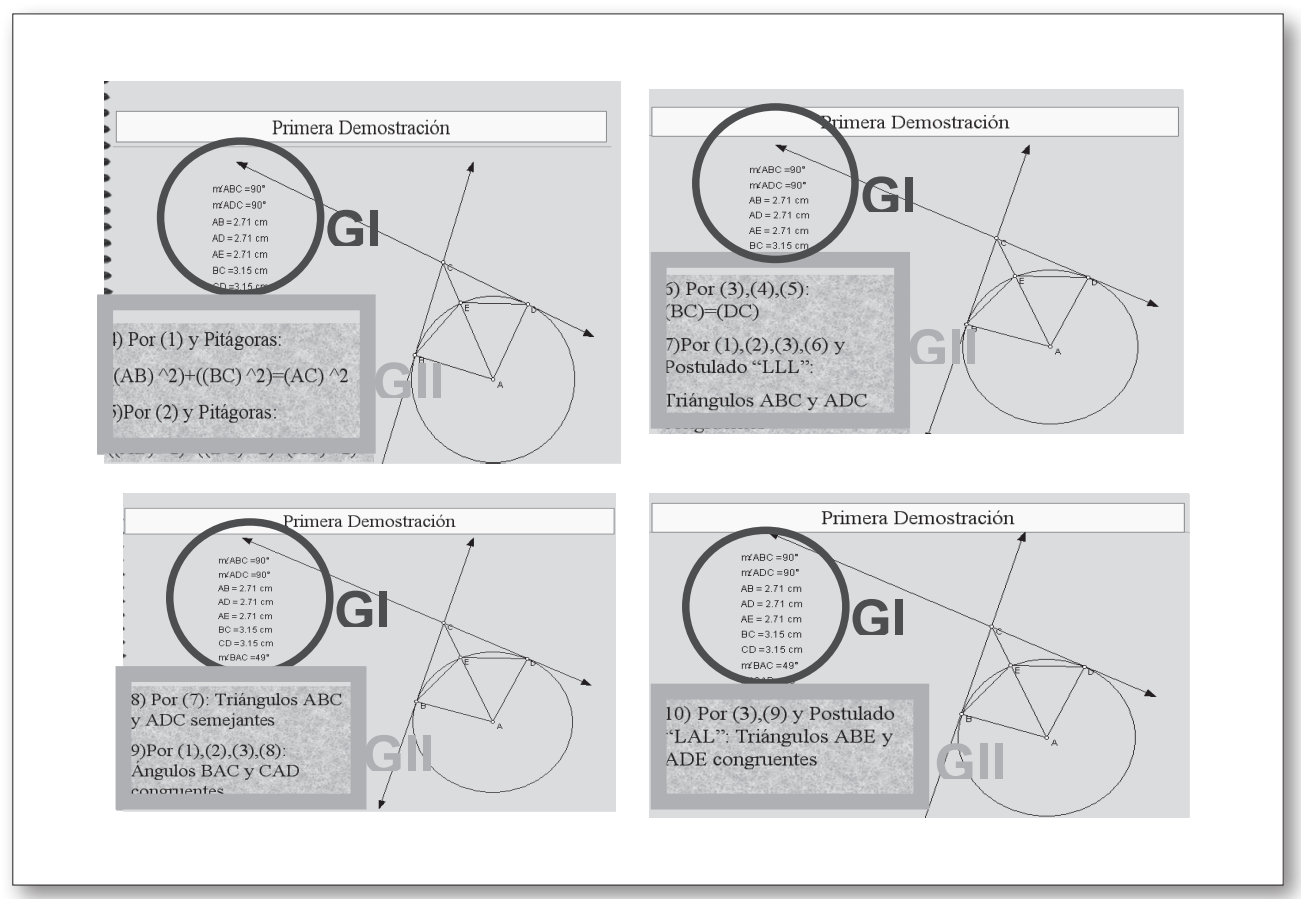

Fig. 5b. Hoja de trabajo proyecto Medida y Demostración.

Podemos afirmar que en el ETG-idóneo del curso de Geometría de primer año cohabitan dos paradigmas geométricos y varían dependiendo de la tarea que se solicita: en las sesiones teóricas, se observan GIII y GII, mientras que en las sesiones prácticas, específicamente en las correspondientes al uso del software, se observa GII yGI.

\section{Aproximación al ETG personal}

En la segunda parte del cuestionario, el objetivo fue conocer el ETG-personal del E-P. Esto se hizo con provocaciones didácticas propuestas de tal forma que ellos fueron situados en dos roles, como estudiante (geómetra) que resuelve un problema, y como profesor (geómetra) que evalúa la producción de alumnos.

Por la evidencia, podemos afirmar que el rol del dibujo es tan fuerte que perturba la elaboración de pruebas y aleja de la concepción teórica (necesaria para responder desde GIII). Las respuestas, en su mayoría, sitúan al E-P (y ahora bajo el rol de profesor) en el paradigma de GI.

Una situación similar ocurre con el uso de los instrumentos geométricos, su «buen uso» es argumento suficiente para conformar una demostración; gran parte de estos E-P afirmó que una prueba -realmente, no demostración- era, o bien estaba cercana de serlo, por el estatus de la construcción como medio de prueba; se trata entonces de una prueba pragmática del tipo empirismo ingenuo y, por lo tanto, en GI. Cabe destacar que 16 de los 18 que respondieron a la pregunta en la que se evidenció la construcción como medio de prueba, efectivamente usaron instrumentos geométricos para seguir la instrucción del argumento de las producciones realizadas. 


\section{El estudiante-profesor bajo el rol de profesor}

A continuación, explicitamos una de las preguntas del cuestionario presentado a 19 E-P, en la que deben producir una respuesta y responder como profesores frente a las eventuales dificultades que puede tener un alumno.

La pregunta 1 que mostramos posee dos provocaciones didácticas: el estudiante debe probar una cuestión perceptivamente cierta en cuanto al paralelismo entre los trazos y además trabajar con una aproximación numérica no necesaria aunque a veces permitida.

Se construye un cuadrado $\mathrm{ABCD}$ de lado $5 \mathrm{~cm}$.

a) Calcular BD.

b) Ubique el punto I del trazo $\mathrm{BD}$ tal que $\mathrm{BI}=2,8 \mathrm{~cm}$, el punto $\mathrm{J}$ del trazo $\mathrm{BC}$ tal que $\mathrm{JC}=3 \mathrm{~cm}$. ¿Es la recta (IJ) paralela a la recta $(\mathrm{DC})$ ?

c) ¿Cuáles son las dificultades o inexactitudes que podrían tener sus futuros alumnos en este ejercicio?

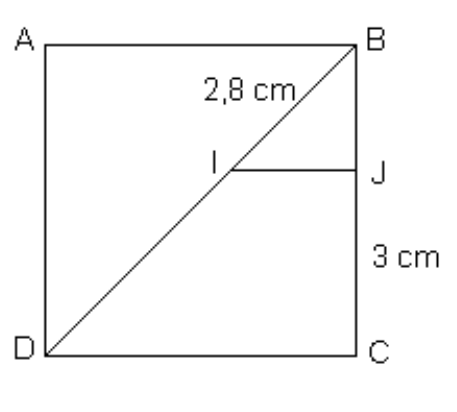

Pregunta 1. Fuente Houdement y Kuzniak (1999).

El a priori de esta tarea fueron cuatro estrategias, incluyendo el uso de instrumentos geométricos dejados expresa y libremente a lo largo del desarrollo del cuestionario. Una de ellas consistía en identificar el triángulo BIJ como rectángulo y usar posteriormente el teorema de Pitágoras, cuyo empleo incorrecto identificamos por efecto de la visualización que interfiere en el referencial. Posteriormente, se utilizan, al igual que en las restantes estrategias, el teorema de Tales y su recíproco. Cada una de estas estrategias se identifica y clasifica en un paradigma geométrico aludiendo o trastocando un polo del ETG.

\section{La articulación de los paradigmas con la tipología de prueba}

Cabe destacar que a nivel de análisis incorporamos una subdivisión en los paradigmas geométricos (Montoya, 2010) inspirados con elementos gravitantes del plano epistemológico: percepción, experimentación y deducción o propiedad, ver tabla 4.

Tabla 4.

Articulación paradigmas con la tipología de prueba

\begin{tabular}{|l|l|l|l|}
\hline \multirow{2}{*}{ Paradigma } & \multicolumn{3}{|c|}{ Cat ETG } \\
\cline { 2 - 4 } & \multicolumn{1}{|c|}{ Experiencia } & \multicolumn{1}{|c|}{ Percepción } & \multicolumn{1}{c|}{ Propiedad } \\
\hline GI & Empirismo ingenuo & Experiencia crucial & Experiencia crucial \\
\hline GII & Ejemplo genérico & Ejemplo genérico & $\begin{array}{l}\text { Experiencia mental } \\
\text { Demostración }\end{array}$ \\
\hline GIII & & Ejemplo genérico & $\begin{array}{l}\text { Experiencia mental } \\
\text { Demostración }\end{array}$ \\
\hline
\end{tabular}


La pregunta 1 está inserta en GII y no esperamos respuestas desde GIII, y en efecto fue lo que sucedió. En 1-a), 18 E-P trabajaron en GII y realizaron pruebas intelectuales: 12 de ellos realizaron experiencia crucial y 6 de ellos, una demostración. En lo que sigue, mostraremos algunas respuestas y posteriormente un resumen de ellas.

\section{GI-exp. y el empirismo ingenuo}

En este grupo clasificamos las respuestas de los estudiantes que usan los instrumentos para medir o constatar vía construcción. Por ello, usamos la notación GI-exp aludiendo a una experimentación en este paradigma. A nivel de pruebas, cabe considerar el objeto particular y manipularlo. Una respuesta representativa de este grupo es la de un estudiante que hace una construcción (hay marcas en la figura) para probar que los trazos en cuestión son paralelos; hay manipulación del objeto geométrico: 3 de las 19 respuestas son de este grupo, a pesar de que son completamente diferentes en cuanto a la existencia del paralelismo: E17, no existe paralelismo con la construcción regla y compás; E18, existe paralelismo, y finalmente E16, no es posible determinar la existencia del paralelismo.

\section{GI-prop. y la experiencia crucial}

En este grupo clasificamos las respuestas de los estudiantes que se apoyan en la dimensión numérica, y responde a la importancia otorgada a la medida de la longitud de un segmento que «debe» aproximarse cuando el número es un irracional. Algunos estudiantes no logran concebir que un número irracional represente la longitud de un segmento visualmente finito. Por ello, usamos la notación GIprop. aludiendo al uso de una propiedad en este paradigma. En 1-b) era necesario utilizar el trazo BD: 5 de los 19 estudiantes aproximan la medida del trazo y cambian de GII a GI. Ellos se apoyan en el marco numérico, aproximan la medida del trazo $\mathrm{BD}=2 \sqrt{5}$ al racional 7,071, con el que las fracciones son equivalentes y así validan el uso del teorema de Tales; luego la respuesta es que hay paralelismo, como muestra el estudiante E12: $\frac{2}{5}=\frac{2,8}{7,071}$

En cuanto a la prueba, no es del todo clasificable en experiencia crucial, pero la dejamos en esta categoría, pues presenta elementos de la figura, de lo que le evoca hacer, es decir, las medidas de los lados se «terminan» $y$ «no pueden» ser representadas por irracionales.

En la tabla 5, se resumen las pruebas realizadas por los estudiantes para 1-b).

Tabla 5.

Resumen de producciones de estudiantes-profesores

\begin{tabular}{|l|l|c|}
\hline Tipo de prueba & Tipo de paradigma & Frecuencia \\
\hline Prueba pragmática: empirismo naïf(ingenuo) & GI-exp. & 3 \\
\hline Prueba pragmática: experiencia crucial & GI-perc. & 4 \\
\hline Prueba pragmática: experiencia crucial & GI-prop. & 5 \\
\hline Prueba intelectual: experiencia mental & GII-prop. & 3 \\
\hline Prueba intelectual: demostración & GII-prop. & 4 \\
\hline Prueba intelectual: ejemplo genérico & GII-perc. & 2 \\
\hline No hay justificación & & 2 \\
\hline & & \\
\hline
\end{tabular}


Se debe aclarar que la suma total sobrepasa el total de estudiantes porque se han clasificado respuestas que se mezclan, es decir, se utilizaron dos pruebas o bien están en un paradigma y buscan información o elementos de otro como en un juego de paradigmas, que abordamos posteriormente.

En 1-c) hay una propuesta para aproximarnos al ETG-personal del E-P en su rol de profesor. En efecto, la mayoría afirma que la dificultad radica en el uso del instrumento geométrico y su imprecisión al confrontar con una respuesta «más teórica» haciendo referencia al uso de teoremas. Mostramos dos respuestas:

E1: Creo que principalmente el alumno es capaz de medir con escuadra la diagonal y le resulta $4,5 \mathrm{~cm}$; sin embargo, teóricamente le da un número distinto por lo que hace que el alumno contraste entre teoría y realidad de sus mediciones.

E17: Las inexactitudes que se pueden dar pueden ser ocasionadas por la imprecisión de los instrumentos, sin embargo, podríamos utilizar el teorema de Tales para comprobar si son o no paralelas.

Desatacamos la respuesta de E1, puesto que para dar la medida de 4,5 cm efectivamente midió la diagonal. En la pregunta1-b), y bajo el rol de estudiante, E17 propone precisamente una respuesta que aborda la construcción con el horizonte de constatar (figura 6).

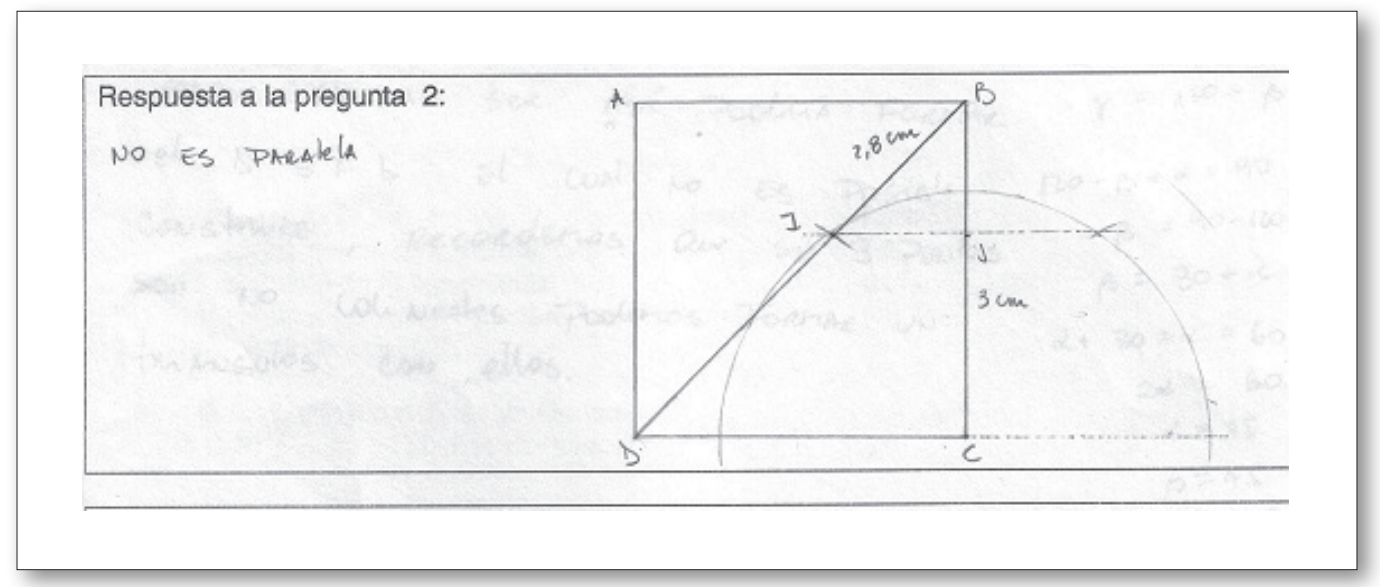

Fig. 6. Respuesta 1-b) del E-P E17 bajo el rol de alumno.

Sin embargo, E17, bajo el bajo el rol de profesor, afirma que hay imprecisión de los instrumentos y propone pasar por el teorema de Tales, confrontando su propia respuesta, su doble rol y evidenciando su propia dificultad (figura 7).

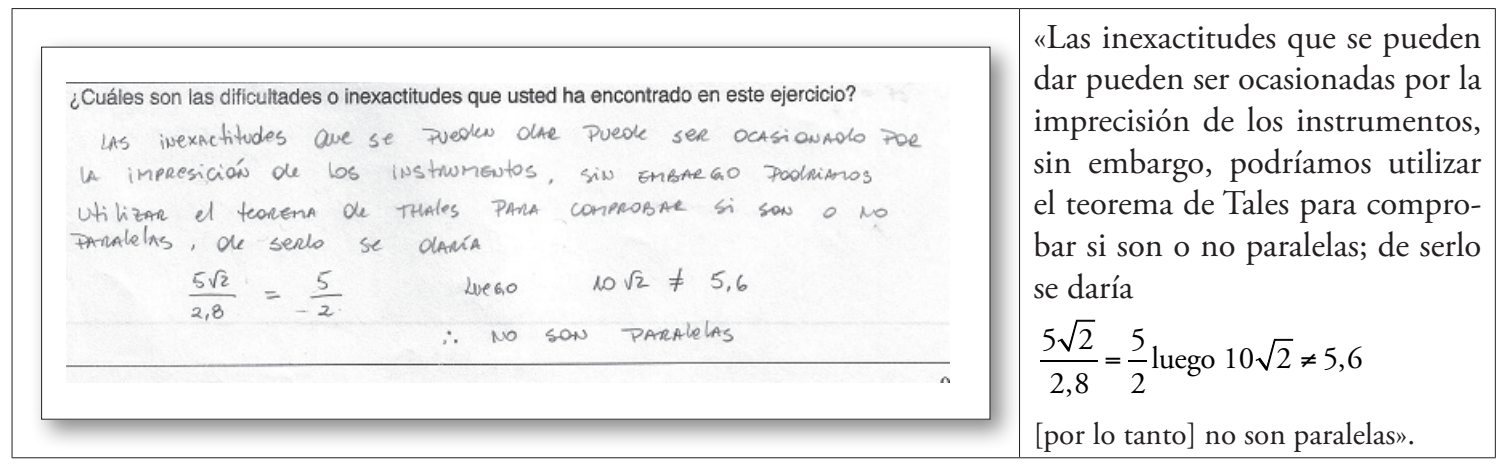

Fig. 7. Respuesta 1-c) del E-P E17 bajo el rol de profesor. 


\section{El juego de paradigmas}

Hay estudiantes que en el momento de realizar una prueba se sitúan en un paradigma y buscan información utilizando elementos característicos de otro; estos elementos son indicadores que diferencian los ETG-personales de estos E-P.

Hay respuestas en las que se busca comprobar con herramientas (instrumentos geométricos) y posteriormente confrontar con un teorema (instrumento teórico). En este caso particular, construyen con el objeto de constatar y con un teorema comprueban que los trazos no son paralelos; es una forma práctica de usar el teorema. Luego estos estudiantes se sitúan en GI y buscan información de GII. En este caso, concebimos un tránsito que anotamos GI/gII, notación que indica la producción en GI que se apoya o se ve influenciada con elementos característicos de GII (usamos notaciones análogas para casos similares).

Hay respuestas de E-P que están en GII en el momento de elaborar la prueba, pero cuando se posicionan como estudiantes toman elementos representativos de GI. En este caso pensamos en GII/gI.

\section{CONCLUSIONES: RUPTURAS Y OBSTÁCULOS}

\section{La estabilidad epistemológica}

En esta investigación, se evidenció que el E-P no es estable en el proceso de prueba en el eje de Geometría; esto es, ante provocaciones didácticas el estudiante (y profesor en formación inicial) mantiene su confusión y reacciona de acuerdo a las exigencias inmediatas (contrato) respondiendo indistintamente en GI y GII. Así, simplemente no enseña a demostrar, no realiza pruebas intelectuales y no es consciente del uso oportuno de las pruebas pragmáticas y de la dificultad que tendrán ellos y sus futuros alumnos al pasar de una prueba pragmática a una intelectual.

En gran parte esto se debe a que el E-P no es sometido a la reflexión explícita de la transposición de la demostración o de otro tipo de argumentación en su formación universitaria, y menos en su desempeño profesional; así lo evidencian sus respuestas a las encuestas a las que fueron sometidos. Ellos mantienen un discurso sobre la importancia de la demostración, pero muestran falta de claridad al responder como geómetra-estudiante y como geómetra-profesor; el rol del dibujo y el uso de instrumentos en sus «demostraciones» lo perturban y lo cambian de paradigma.

En términos del marco utilizado en este estudio, afirmamos que ETG-personal es inestable ya que el proceso de prueba del plano cognitivo está fuertemente tensionado por la visualización.

\section{Las rupturas}

La investigación da cuenta de rupturas y obstáculos que se hacen presentes en los pasajes institucionales que vive el E-P entre la institución formadora y el liceo, por el cambio del plano epistemológico del ETG que debe desplegar en ambas instituciones. La ruptura de carácter didáctico en el primer pasaje (liceo-universidad) se debe a la concepción de demostración de los estudiantes que se enfrentan a cambios de pruebas pragmáticas a intelectuales. En el segundo pasaje (universidad-liceo), el E-P se ve enfrentado a enseñar la demostración en Geometría sin haber sido formado de manera expresa para realizar este tipo de tareas con sus futuros alumnos; su ETG-idóneo no está potenciado por la formación inicial, y queda más bien sujeto a su ETG-personal, que como dijimos transita entre GI y GII.

En cuanto a la institución formadora (universidad), el estudiante aprende a demostrar sin que se haya sometido a un proceso explícito de aprendizaje. Los formadores universitarios exhiben y exigen la demostración como una herramienta del matemático. La universidad no siempre incentiva a los 
estudiantes a reflexionar (explícitamente) sobre la transposición de la enseñanza de la demostración ni de ningún otro tipo de prueba exigible y/o viable en la institución liceo.

Por otro lado, se evidencia que en los programas oficiales escolares la concepción de demostración no es única, esto es, hay pruebas pragmáticas aceptadas como demostración. Similar situación se evidencia en textos escolares, en los que además en las pocas ocasiones que se exhiben pruebas de una propiedad en Geometría, las pragmáticas se privilegian por encima de las intelectuales.

Al considerar estas rupturas en ambas instituciones, levantamos la hipótesis de que el profesor debutante se puede dejar influenciar por una de las concepciones de demostración antes presentadas, lo que a su vez implicaría que los alumnos se vean sometidos a un solo paradigma y ETG.

Por otro lado, respecto a la ruptura que hace referencia a la presencia del obstáculo cognitivo, (esto es, la dificultad en el traspaso de pruebas pragmáticas a intelectuales por un lado, y por otro, la dificultad de expresar argumentaciones del oral al escrito), pudimos evidenciar que en la articulación de los planos (figura 1) la génesis discursiva está debilitada por el rol gravitante que juega el dibujo en las producciones (génesis figural), perturbando el ETG.

Los propios estudiantes reconocen que redactar una justificación es más complejo que entender una demostración; el de primer año identifica los «pasos» de una demostración como una verdad, pero estas «verdades» no son integradas en un sistema lógico complejo, sino que en definitiva la comprensión de una demostración se reduce a entender «cada una» de esas verdades.

\section{Aportes y consideraciones finales}

Consideramos relevante, a nivel de formación, que los E-P tengan la oportunidad de explotar al máximo los ETG transitando expresamente con tareas entre uno y otro paradigma. Esto permite tener mayor claridad en el momento de enseñar Geometría: se puede utilizar un paradigma apropiado a cada nivel escolar al potenciar armónicamente los polos del ETG y no abusar, por ejemplo, del polo de visualización; también se puede fomentar la circulación entre el polo prueba y el referencial, al potenciar una economía matemática (redacción, formalismo e implicaciones pulcras) propia del referencial que se desea enseñar.

Otro elemento importante que debe ser abordado por los formadores universitarios es el rol de la figura geométrica en el proceso de prueba. Si los E-P tienen claridad de que una figura geométrica tiene distintos estatus en diferentes pruebas, podrán contar con elementos para el proceso transpositivo de la demostración en Geometría con sus futuros alumnos.

En este trabajo estudiamos la formación en Geometría de los futuros profesores en una universidad en particular y hemos constatado que la demostración es la prueba privilegiada por la institución formadora. Este razonamiento de validación pasa a ser un conocimiento adquirido que podría utilizar y exigir en su desempeño profesional o no hacerlo, porque no tiene las herramientas para hacer una transposición de este saber.

La investigación está basada en la tesis doctoral de la autora bajo la dirección de Alain Kuzniak, y en el trabajo de un equipo de investigación que estudia a profesores debutantes (Fondecyt, 1110988) en cuanto a su estabilidad paradigmática y que propone un $\mathrm{ETM}_{\mathrm{A}}$ en Álgebra. 


\section{REFERENCIAS BIBLIOGRÁFICAS}

Araya, R. (2008). Saber Pedagógico y Conocimiento de la Disciplina Matemática en Profesores de Educación General Básica. (Informe de investigación). Departamento de Estudios y Desarrollo. División de Planificación y Presupuesto, Ministerio de Educación, Gobierno de Chile.

ArsaC, G. (1987). L’origine de la démonstration: Essai d'épistémologie didactique. Recherches en didactique de la mathématique, 8(3), pp. 267-312.

Balacheff, N. (1987). Processus de preuve et situations de validation. Educational Studies in Mathematics, 18(2), pp. 147-176.

Barbine, E. (1989). Trois démonstrations pour un théorème élémentaire de géométrie sen de la démonstration et de la géométrie. En La démonstration mathématique dans l'histoire. Besançon, France: Institute de Recherche sur l'Enseignement des Mathématiques de Besançon et Lyon, pp. 57-79.

Boero, P. (1999). Argumentación y Demostración: Una relación compleja, productiva e inevitable en las matemáticas y en la educación matemática. Disponible en línea: <http://www-didactique.imag. fr/preuve/Newsletter/990708Theme/990708ThemeES.html>.

Brousseau, G. (1983). Les obstacles épistémologiques et les problèmes en mathématiques. Recherches en Didactique des Mathématiques, 4(2), pp. 165-198.

Brousseau, G. (1998). Théorie des Situations didactique: didactique des mathématiques. Grenoble, France: La Pensée Sauvage.

Castela, C. (2005). A propósito de los conocimientos que no se enseñan explícitamente aunque son necesarios para tener éxito en las matemáticas escolares. Revista Latinoamericana en Matemática Educativa, 8(2), pp. 111-127.

Chevallard, Y. (1985). La transposition Didactique : du savoir savant au savoir enseigné. Grenoble, France: La Pensée Sauvage.

De Villiers, M. (1993). El papel y la función de la demostración en matemática. (José Álvarez, trad.). Revista Epsilon, 23, pp. 15-30. (Obra original publicada en 1990). Disponible en línea <http:// mzone.mweb.co.za/residents/profmd/proofb.pdf>.

Ducrot, O. (1972). Dire et ne pas dire. Paris, France: Hermann.

Durand-Guerrier, V. y Arsac, G. (2003). Méthodes de raisonnement et leurs modélisations logiques. Spécificité de l'analyse. Quelles implications didactiques ? Recherches en Didactique des Mathématiques, 23(3), pp. 295-342.

Duval, R. (1991). Structure du raisonnement déductif et apprentissage de la démonstration. Educational Studies in Mathematics, 22(3), pp. 233-261.

Duval, R. (1992). Argumenter, Démontrer, Expliquer: continuité ou rupture cognitive? Revue Petit $x, 31$, pp. 37-61.

Duval, R. (1995). Sémiosis et pensée humaine. Berne, Suisse: Peter Lang.

Godino, J. y Recio, Á. (2001) Significados institucionales de la demostración. Implicaciones para la educación matemática. Revista Enseñanza de las Ciencias, 19(3), pp. 405-414.

Gueudet, G. (2005). Thème 3-La transition secondaire-supérieur. En A. Rouchier e I. Bloch (Eds). Perspectives en didactique des mathématiques: Cours de la XIII ème École d'été de didactique de mathématiques.Grenoble, France: La Pensée Sauvage, pp. 159-176.

Hanna, G. (1995). Challenges to the importance of proof. For the Learning of Mathematics, 15 (3), pp. $42-49$

Hanna, G. (2000). Proof, Explanation and Exploration: an overview. Educational Studies in Mathematics, 44, pp. 5-23.

http://dx.doi.org/10.1023/A:1012737223465 
Hanna, G. y Sidoli, N. (2007). Visualisation and proof: a brie survey of philosophical perspectives. Mathematics Education, 39, pp. 73-78.

Henríquez, C. (2009). Semejanza de Figuras Planas en dos Textos Escolares: Tipo de geometría y el razonamiento del Espacio de Trabajo Geométrico. Trabajo final Magister (no publicada). Pontificia Universidad Católica de Valparaíso, Chile.

Hersh, R. (1993). Proving is convincing and explaining. Educational Studies in Mathematics, 24, pp. 389-399.

http://dx.doi.org/10.1007/BF01273372

Houdement, C. y Kuzniak, A. (1999). Un exemple de cadre conceptual pour l'étude de l'enseignement de la géométrie en formations des maîtres. Educational Studies in Mathematics, 40, pp. 283-312.

Houdement, C. y Kuzniak, A. (2000). Formation des maîtres et paradigmes géométriques. Recherches en Didactique des Mathématiques, 20(1), pp. 89-116.

http://dx.doi.org/10.1023/A:1003851228212

Houdement, C. y Kuzniak, A. (2006). Paradigmes géométriques et enseignement de la géométrie. Annales de Didactique des mathématiques et des sciences cognitives, 11, pp. 175-216.

Kunn, T.S. (1962/1971). La estructura de las revoluciones cientificas (Agustín Contín, trad.). México: Fondo de Cultura Económica.

Kuzniak, A. (2004). Paradigmes et espaces de travail géométriques. (Note pour l'habilitation à diriger des recherches). Paris, France : Institute de Recherche sur l'Enseignement des Mathématiques Paris VII.

Kuzniak, A. (2011). L’espace de travail mathématique et ses génèses. Annales de Didactique et de Sciences Cognitives, 16, pp. 9-24.

Menares, R. (2009). Estudio del Trabajo Geométrico, Tipos de Argumentaciones y Demostración en Geometría: la Mirada al Profesor. Trabajo final Magister (no publicada). Pontificia Universidad Católica de Valparaíso, Chile.

MINEDUC. (2009). Programas oficiales de educación matemática. Ministerio de Educación, Gobierno de Chile. Disponible en línea: $<$ http://www.mineduc.cl/index5_int.php?id_portal=47\&id_ contenido $=17116 \&$ id_seccion $=3264 \& \mathrm{c}=1>$.

Montoya, E. (2010). Étude de la transformation des connaissances géométriques dans la formation universitaire des professeurs de lycée de mathématiques au Chili. (Thèse de doctorat). Université Denis Diderot, Paris, Francia.

Plantin, C. (1996). L'argumentation. Paris, Fance: Éd. du Seuil.

SIMCE (2003). Ministerio de Educación Chile. Unidad de Currículum y Evaluación. Resultados Nacionales Sistema de Medición de la Calidad de la Educación. Santiago, Chile.

SIMCE (2007). Ministerio de Educación Chile. Unidad de Currículum y Evaluación. Resultados Nacionales Sistema de Medición de la Calidad de la Educación. Santiago, Chile.

SIMCE (2009). Ministerio de Educación Chile. Unidad de Currículum y Evaluación. Resultados Nacionales Sistema de Medición de la Calidad de la Educación. Santiago, Chile. Disponible en línea:<http:/www.agenciaeducacion.cl/wp-content/files_mf/informenacionalderesultadossimce2010247mb.pdf>.

Toulmin, S.E. (1958). The Uses of Argument. Cambridge, UK: University Press. 


\title{
The process of proof in the geometric work space: initial training teachers
}

\author{
Elizabeth Montoya Delgadillo \\ Instituto de Matemática \\ Pontificia Universidad Católica de Valparaíso, Chile \\ emontoya@ucv.cl
}

In the university, the proof is often considered a natural practice of students in Mathematics; because it is a working tool for the students and they use it in their courses according to typical characteristics from different mathematical theories such as techniques, language, rigor, and so on. It happens that, in the universities, the proof is considered a practice and it is not explicitly taught and therefore becomes a "paramathématique" notion (Chevallard, 1985). In high school, it has the status of a mathematical notion and the teacher has to teach, make and ask it to their students. Then, the question is how a novel teacher can transpose this knowledge if he has not had this process in their initial training.

Our objective was to study the joint that a novel teacher does by facing a "target of teaching in terms of taught knowledge" (ibid) about the proving process where the proof is inserted (Balacheff, 1987). Since the transposition of knowledge is performed after the initial training, we study the dual role and passage between two educational institutions: the first one, from a high school student to a college student, and the second one, the latter to a secondary school teacher.

In this article, we present a research about nineteen students who work as student-teachers, E-P, which are high school teachers and college students; this dual role is enabling us to know the status conferred to the required and made proof in Geometry. We postulate three ruptures, core idea of our research, on teaching and learning proofs in Geometry, coming from epistemological, didactic and cognitive obstacles (Brousseau, 1998) respectively.

Here we use the Theory of Paradigms and Geometric Work Space, ETG, by Houdement \& Kuzniak (1999, 2000, 2006) and (Kuzniak, 2004) to analyze the novel Chilean teachers and the obstacles that are generated because of the roles and status of the proof in high school institutions that differ from those in the university. At present, the theory is considered a global Mathematical work space, ETM, which depends on the mathematical field, e.g., the ETG is now $\mathrm{ETM}_{\mathrm{G}}, \mathrm{ETM}_{\mathrm{A}}$ will be for analysis, et cetera, and paradigms can be interpreted as the characterization of the ETM (Kuzniak, 2011).

In this research evidence, it becomes clear that the E-P is not stable in the proving process in Geometry, that is, under the didactic provocations the E-P remains confused and reacts according to the immediate demands (contract) between paradigms from either GI or GII. Then, he does not teach to prove, does not do intellectual proofs, and is not aware of the appropriate use of pragmatic trials and the difficulties that he and their future students will have to move from a pragmatic proof to a intellectual one (Balacheff, 1987).

Overall, the E-P does not face a reflection of the explicit transposition of the proof or another type of argument in the university training and neither their professional performance; as it was well evidenced by their responses to the surveys submitted. They hold a speech on the importance of the proof, but they show a lack of clarity when responding as a geometric-student and as a geometric-teacher; the role of the drawing and the use of instruments in their proofs disrupt them and change the paradigm.

In terms of the theory, this study asserts that personal ETG is unstable because the proving process of the cognitive plane is heavily pressured by visualization.

This qualitative research gives evidence that at university, the student learns to show without having undergone an explicit learning process. The university teachers exhibit and require the proof as a mathematical tool. The university does not always encourage students to (explicitly) reflect on the teaching transposition of the proof or any other type of required and feasible proving in high school institutions. 
